\title{
Neuronal processing of behaviourally generated optic flow: experiments and model simulations
}

\author{
Roland Kern, Maik Lutterklas, Christian Petereit, Jens P Lindemann \\ and Martin Egelhaaf \\ Lehrstuhl für Neurobiologie, Fakultät für Biologie, Universität Bielefeld, Postfach 100131, \\ D-33501 Bielefeld, Germany \\ E-mail: roland.kern@biologie.uni-bielefeld.de
}

Received 4 December 2000

\begin{abstract}
The stimuli traditionally used for analysing visual information processing are much simpler than what an animal sees when moving in its natural environment. Therefore, we analysed in a previous study the performance of an identified neuron in the optomotor system of the fly by using as visual stimuli image sequences that were experienced by the animal while walking in a structured environment. These electrophysiological experiments revealed that the fly visual system computes from behaviourally generated optic flow a rather unambiguous representation of the animal's self-motion. In contrast to conclusions based on simple stimuli, the directions of turns are represented by an interneuron, the HSE cell, quite independent of the spatial layout of the environment and its textural properties when the cell is stimulated with behaviourally generated optic flow. This conclusion is substantiated here by further experimental evidence. Moreover, it is shown that the largely unambiguous responses of the HSE cell to behaviourally generated optic flow can be replicated to a large extent by a network model of the fly's visual motion pathway. These results stress the significance of naturalistic stimuli for analysing what is encoded by neuronal circuits under natural operating conditions.
\end{abstract}

\section{Introduction}

Global retinal image displacements experienced when moving through a structured environment ('optic flow') are a rich source of information that is exploited efficiently by animals and humans to guide their locomotion (Lappe 2000, Miles and Wallman 1993, Gibson 1979). Accordingly, neurons have been found in a variety of animals which are sensitive to different aspects of optic flow as experienced, for instance, when the animal turns about a particular body axis, when it translates in a certain direction or when it approaches an obstacle (see reviews by Lappe (2000) and Miles and Wallman (1993)). 
When the animal turns on the spot, the velocities of the retinal image displacements are independent of the three-dimensional layout of the environment. However, during any realistic locomotion, i.e. during movements which contain a translatory component, the optic flow is much affected by the distance between the objects in the environment and the animal (Koenderink 1986). Optic flow, thus, contains information about both the animal's selfmotion and the environment, although in a highly ambiguous way. Theoretical solutions to disambiguate the superimposed information exist (Dahmen et al 1997). Moreover, honeybees could be shown in behavioural experiments to compute unambiguous information about the retinal velocities of their environment while flying freely in textured flight arenas (for a review see Srinivasan et al 1999). Nevertheless, responses of motion sensitive neurons in a large variety of animal groups were found to depend not only on velocity but also on various other stimulus parameters, such as the contrast and the texture of the stimulus pattern (see e.g. for insects, Eckert 1980, for birds, Wolf-Oberhollenzer and Kirschfeld 1990, for mammals, Cassanello et al 2000, Baker 1990, Pollen et al 1978). Hence, the responses of these neurons are highly ambiguous with respect to velocity encoding. It should be noted, however, that most inferences about what motion sensitive neurons represent are based on motion stimuli which were designed for analytical purposes and are simpler than the optic flow an animal encounters in behavioural situations.

Given these ambiguities in neuronal responses it came as a surprise that a motion sensitive neuron in the fly optomotor system appears to encode the direction of self-motion largely independent of the properties of the environment when stimulated by naturalistic optic flow (Kern et al 2001). In contrast to other recent studies where visual interneurons were stimulated by image sequences that an animal might have experienced on artificial tracks of locomotion that were designed by the experimenter (Kern et al 2000, Mulligan et al 1997, Kim et al 1997, Pekel et al 1996), we used for stimulation optic flow that was actually experienced by the animal while walking about in a three-dimensional environment. The neural representation of behaviourally generated retinal image sequences has been studied before in Limulus, where optic nerve recordings were made in almost freely walking animals (Passaglia et al 1997) as well as in models of visual systems (Kording et al 2000, Passaglia et al 1997).

In our analysis spontaneous walking behaviour was monitored by a video camera and the retinal image sequences experienced during locomotion were reconstructed. These behaviourally generated image sequences were replayed in electrophysiological experiments to an identified interneuron in the fly's third visual neuropile, the HSE cell (figure 1). The HSE cell (Hausen 1982a, b) is one of the so-called tangential cells (TCs) and is thought to play an important role in mediating optomotor course control (for reviews see Egelhaaf and Borst 1993a, Hausen and Egelhaaf 1989, Egelhaaf et al 1988). The HSE cell pools with its large dendritic tree the outputs of many retinotopically organized local motion sensitive elements and responds to visual motion with graded membrane potential changes which may be superimposed by rapid spikelike depolarizations. The preferred directions of the local input elements vary in a way that can be interpreted as an adaptation to detect the visual consequences of rotations about the vertical body axis (Krapp 2000). The specificity of the HSE cell for rotational optic flow is further enhanced by synaptic input from the contralateral eye (Krapp et al 2001, Horstmann et al 2000, Hausen 1982b). Despite its sophisticated input organization the HSE cell does not exclusively respond to the rotational component of optic flow. Rather it also responds strongly to simple approximations of translational optic flow and even monocular motion (Kern and Egelhaaf 2000, Hausen 1982b) as well as to purely translatory motion in a three-dimensional environment (Kern et al 2001). Moreover, the response amplitude of the HSE cell was found to depend in a nonlinear way on stimulus parameters, such as velocity, contrast and the textural properties of the stimulus patterns (Haag and Borst 1997, Egelhaaf 


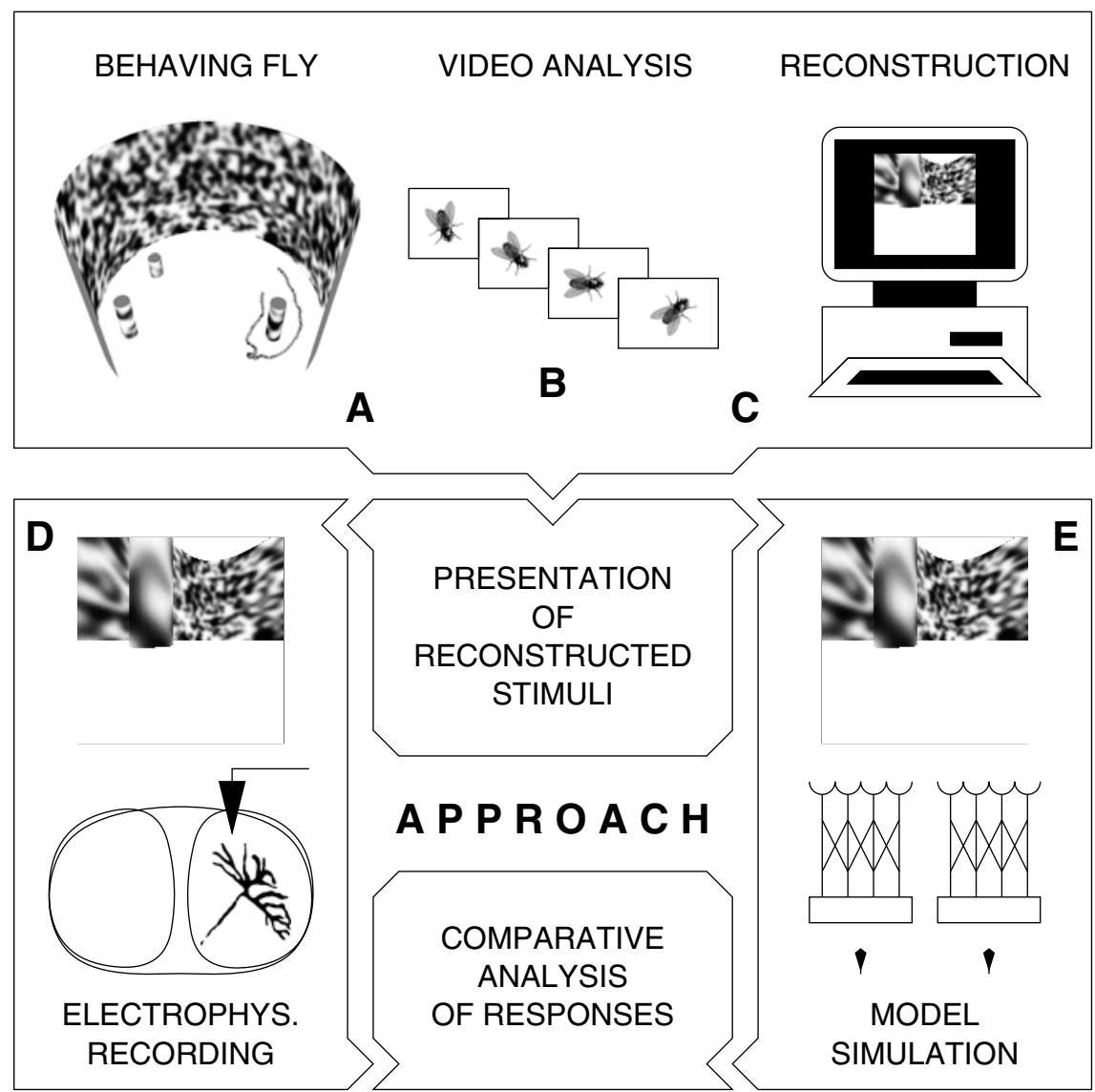

Figure 1. Approach of the present study. (A) Flies walking freely in an arena are video recorded. (B) The video is grabbed off-line frame by frame and the position and orientation of the fly are determined in each digitized video frame. (C) The trajectory data are used to control the path of a simulated camera in a virtual three-dimensional environment that mimics the arena. The size of the field of view of the camera is adjusted to match the receptive field properties of the cell to record from in subsequent electrophysiological experiments. (D) In the electrophysiological experiments the cell monitored is stimulated by the sequence of reconstructed images. (E) The same images are fed into a model of the fly's motion pathway (details shown in figure 2). The local motion computations as well as the spatial integration of the local motion signals are indicated schematically for the left and right halves of the model visual system. Finally, the output of the HSE cell and its model equivalent are compared.

and Reichardt 1987, Hausen 1982a, b). From these findings, we expected the responses of the HSE cell to behaviourally generated optic flow to provide only highly ambiguous information about the animal's self-motion. In contrast to these expectations, we could show that the actual responses of the HSE cell encode the turning direction of the animal largely independent of the spatial layout of the environment, its textural properties, and the translatory component of the optic flow (Kern et al 2001).

In this account we will substantiate this conclusion on an extended database and demonstrate by computer simulations that these results can be explained to a large extent on the basis of a network model of the fly's motion pathway. 


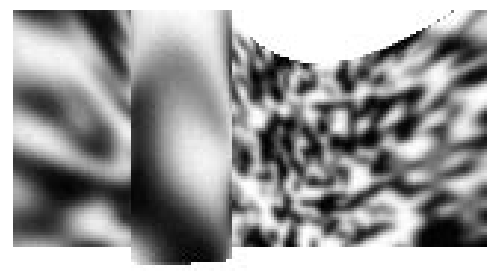

retinal

input

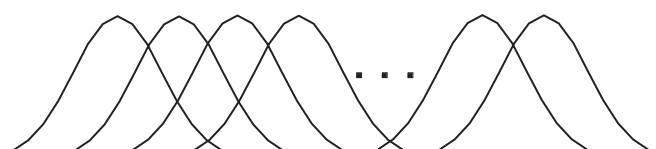

spatial

filtering

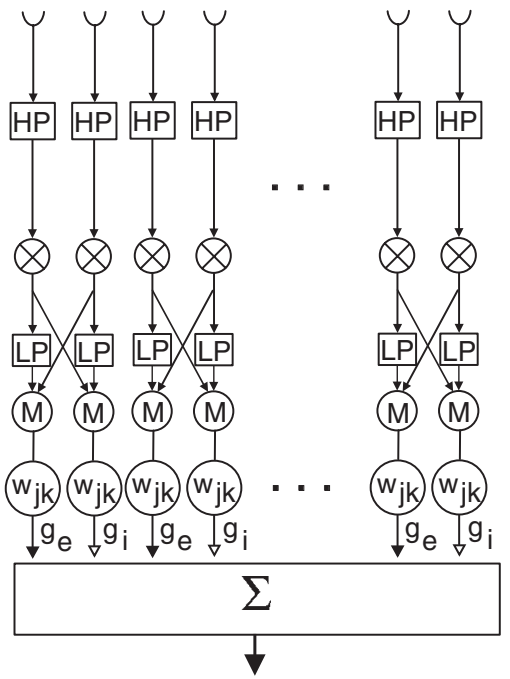

temporal

filtering

local

motion

detection

nonlinear

integration

Figure 2. Schematic diagram of a model used as a first approximation to explain the processing of motion information in the fly's visual system. For the sake of clarity only one horizontal dimension of the model is sketched. The 62 retinotopically organized horizontal elements are replicated 61 times in the vertical dimension. There are three major processing stages (peripheral prefiltering, local motion detection and nonlinear integration of local motion signals). The parameter settings and computations performed at the different processing stages are explained in section 2. Abbreviations: HP, temporal first-order high-pass filter; LP, temporal first-order lowpass filter; $\mathbf{M}$, multiplication stage of the elementary movement detector; $w_{j k}$, constant weight factors which adjust the spatial sensitivity distribution of the model to that of the HSE cell; $g_{\mathrm{e}}, g_{\mathrm{i}}$, gain factors of the excitatory and inhibitory output channels of the elementary movement detectors, controlling excitatory and inhibitory conductances; $\Sigma$, nonlinear integration of the local motion signals.

\section{Methods}

\subsection{Dissection of the animals and electrophysiological recording}

Female flies of the genus Lucilia were used in all experiments. The animals were bred in our laboratory culture. To avoid in-breeding, the culture was refreshed several times a year with animals caught in the wild. For a description of the dissection of the animals as well as the intracellular recording procedure see our previous account on the neuronal representation of behaviourally generated optic flow (Kern et al 2001). Signals were sampled and stored at 
a rate of $2 \mathrm{kHz}$ using programs written in ASYST (Keithley Instruments) and downsampled afterwards to different extents as specified in the figure legends. The HSE cell was identified on the basis of physiological criteria, i.e. by its response mode, its preferred direction of motion in the contralateral and ipsilateral visual fields, and the location of the receptive field (Hausen 1982a, b).

\subsection{Generation of visual stimuli}

Image sequences as experienced by walking flies were obtained by the following procedure. Flies walking in a circular arena were video-recorded $(50 \mathrm{~Hz})$. The walls of the arena were covered with random textures (see figures $1,2,4$ ) while the floor was homogeneously white. The arena was illuminated indirectly from above (luminance, $210 \mathrm{~cd} \mathrm{~m}^{-2}$ at the floor in the centre of the arena). Various textured objects were introduced into the arena. Two arena sizes were used (diameters 0.5 and $0.31 \mathrm{~m}$, height $0.3 \mathrm{~m}$ ). The video sequences were digitized. The position and the orientation of the body axes of the flies were automatically detected in each frame by specifically designed software. From the parameters of locomotion the retinal projection of a reconstruction of the arena was computed using a virtual reality software (RealAx Software, Karlsruhe, Germany). The retinal images were computed after interpolating one position and orientation between subsequent positions and orientations of the fly along its walking track and after smoothing of the time-dependent position and orientation traces. In this way 100 images per second were calculated for each filtered track. This procedure was required because of the high temporal resolution of the fly's eye.

The interpolation and smoothing of the data were done in the following way. Intermediate positions between those determined in subsequent frames were linearly interpolated. The corresponding orientations were linearly interpolated on the basis of their sine and cosine components. In this way position and orientation parameters were available at intervals of $10 \mathrm{~ms}$. The position traces were filtered by a triangular filter with a width of $50 \mathrm{~ms}$ to eliminate position jitter resulting from methodological inaccuracies. This procedure did not have any major functional significance, because the position jitter did not much affect the corresponding retinal images. The time-dependent orientations of the fly's body axis were filtered on the basis of their sine and cosine components by a triangular filter with a width of $130 \mathrm{~ms}$. The time constant of this filter was motivated by the specific walking mode of flies. Flies were shown in an analysis of their walking behaviour employing high-speed film techniques to oscillate around their direction of propagation with every step cycle (Strauss and Heisenberg 1990). Since these oscillations of the body axis in a frequency range around $10 \mathrm{~Hz}$ are largely compensated for by head movements, they are believed to induce only negligible image displacements (Strauss and Heisenberg 1990). Hence our filtering of the time-dependent orientation traces led to a reasonable approximation of the optic flow on the eyes of walking flies.

Image sequences were reconstructed for the original walking trajectory and the original visual surround as well as for manipulated versions of both of them. The various manipulations that were used are specified in the corresponding figure legends. Manipulations were done in a defined way in order to investigate in electrophysiological experiments ('replay experiments') the cell's response specificity to particular optic flow components. The image sequences were presented on a 15-inch computer monitor with a video player at a rate of 100 images per second (luminance of darkest pixels $0.1 \mathrm{~cd} \mathrm{~m}^{-2}$, of brightest pixels $61.4 \mathrm{~cd} \mathrm{~m}^{-2} ; 64$ brightness levels). At this presentation rate no motion aliasing occured even at the highest angular velocities of the animal. The image size was $\pm 60^{\circ}$ in azimuth and $\pm 60^{\circ}$ in elevation with $0^{\circ} / 0^{\circ}$ corresponding to the frontal midline of the animal. The image thus covered most of the receptive field of the HSE cell (Hausen 1982b). 
Although all electrophysiological experiments were performed on the HSE cell in the righthand half of the brain, the responses of the contralateral HSE cell were indirectly inferred. This was done by presenting to the right-hand HSE cell images that were mirror-inverted about their vertical midline.

\subsection{Experimental procedure and data analysis}

Image sequences were presented in pseudorandom order with a $10 \mathrm{~s}$ interstimulus interval. The first image of a sequence was presented for $1 \mathrm{~s}$ before motion started. The average membrane potential in the last $250 \mathrm{~ms}$ of this period was taken as a reference level. All responses are given with respect to this level, which varied between cells and image sequences from -40 to $-48 \mathrm{mV}$. In different cells different numbers of response traces could be obtained. Average responses to a given stimulus were determined by first averaging over all individual response traces of each cell and subsequent averaging over these mean responses of all cells.

Differences between responses elicited by the original and by a manipulated stimulus may have two sources. (i) They may be attributable to the different stimuli. (ii) They may be due to neuronal variability. To disambiguate these two sources, the similarity of responses to different stimuli was related to the similarity of responses to identical stimulation. The similarity index is given by the ratio of the peak of the normalized cross-correlation of individual responses between stimulus classes to the peak of the normalized cross-correlation of responses within a class. The similarity index was first determined for each cell and then averaged over cells. Note that a similarity index of unity may also be obtained if the responses elicited by the original and the manipulated stimulus have an identical time course but differ in their amplitude. Therefore, we analysed whether the responses to the two different stimuli fluctuate over time with approximately the same amplitude. This was done by first averaging and filtering (rectangular filter, width $30 \mathrm{~ms}$ ) the individual responses to a given stimulus. Then the standard deviation over time was calculated for each average response. Finally, the ratio between the standard deviations of the responses to the manipulated and the original version of the stimulus was calculated. Data analysis was performed with Matlab5.3 (MathWorks, Natick, MA).

\subsection{Model simulations}

Our model is an elaboration of models previously established to explain various aspects of motion detection by the fly visual system. It is a hybrid of algorithmic components and components which represent a simple equivalent circuit of a nerve cell. The peripheral part of the motion detection pathway, i.e. the peripheral filters corresponding to the retina and the first visual neuropile, as well as the motion detection process itself, were modelled by linear filters, the outputs of which interact with each other by simple mathematical operations. Hence, although the output of this part of the model was meant to fit the output of the local motion detectors of the fly, it was not intended to approximate the cellular operations in detail. Spatial pooling of the many local motion detectors was achieved by means of a simple equivalent circuit of a patch of membrane, where the positive and negative outputs of the local motion detectors controlled excitatory and inhibitory conductances, respectively. The model output thus can be interpreted as representing the graded postsynaptic potential of the HSE cell.

The model consists of three processing stages, which will be referred to as (i) peripheral filtering, (ii) local motion detection and (iii) spatial pooling of local motion information (figure 2). The simulations were performed at a temporal resolution of $100 \mathrm{~Hz}$, i.e. at the rate at which images were presented in the electrophysiological experiments. Part of the model 
simulations were repeated at a temporal resolution of $400 \mathrm{~Hz}$. The high temporal resolution did not much affect the response traces. Hence, there are no indications that the $100 \mathrm{~Hz}$ resolution induced artefacts. The left and the right HSE cells were modelled separately. The size of the input frames was $320 \times 320$ pixels, corresponding to a visual field of either eye of $80^{\circ}$ by $80^{\circ}$, including $18.75^{\circ}$ horizontal binocular overlap.

Peripheral filtering started with spatial downsampling of each input frame to an equidistant retinal receptor matrix consisting of $62 \times 62$ elements according to the experimentally established spatial sensitivity distribution of the individual photoreceptors (Smakman et al 1984) by a two-dimensional Gaussian $\left(\sigma=0.75^{\circ}\right)$. The receptor distance was 5 pixels with respect to the input frame and thus amounted to $1.25^{\circ}$. There was a zone of binocular overlap in the frontal visual field extending for each eye by $18.75^{\circ}$ into the contralateral visual field. To mimic the phasic response properties of output cells of the fly's first visual neuropile (Juusola et al 1996, Laughlin 1994), the photoreceptor output was temporally filtered with a firstorder high-pass filter (time constant $35 \mathrm{~ms}$ ). The resulting signals were fed into correlationtype motion detectors, which have been shown to explain many aspects of the local motion detector (EMD) responses in the fly's visual system (for a review see Egelhaaf and Borst 1993b). Each detector consisted of two mirror-symmetrical subunits (figure 2). The subunits received their inputs from two input channels that were neighbours along the horizontal axis of the model retina. In each subunit the signal was temporally filtered with a first-order low-pass filter (time constant $150 \mathrm{~ms}$ ) and multiplied by the unfiltered signal of the neighbouring input. The model HSE cell was excited by one and inhibited by the other subunit output. The subunit outputs were assumed to modulate the membrane conductances $\left(g_{\mathrm{e}}, g_{\mathrm{i}}\right)$ in the model HSE cell with reversal potentials $E_{\mathrm{e}}>0$ and $E_{\mathrm{i}}<0$, respectively. Zero corresponded to the resting potential of the cell. The membrane conductances introduced a nonlinearity into the model HSE cell, which was partly responsible for the so-called gain control of the HSE cell. By 'gain control' we mean that the responses of the HSE cell are largely invariant against changes in pattern size, while still depending on pattern velocity (Single et al 1997, Borst et al 1995, Hausen 1982b). The specific gain control properties are determined by the relation between the cell's leak conductance $\left(g_{0}\right)$ and the membrane conductances $\left(g_{\mathrm{e}}, g_{\mathrm{i}}\right)$ that are modulated by the visual input. To adjust the spatially distributed motion detector input of the model HSE cell to the spatial sensitivity distribution of the HSE cell (Hausen 1982b), the subunit outputs were weighted according to their position within the two-dimensional detector array. We used a two-dimensional phase-shifted spatial $\cos ^{2}$ function for the approximation of the sensitivity distribution. The local sensitivities $w_{j k}$ ( $j=$ azimuthal index, $k=$ elevational index, with $j, k=[0, \ldots 61]$; for each eye $j=0$ corresponds to its left margin) amounted to

left eye $: w_{j k}=\cos ^{2}((0.16)(2 \pi / 62)(j-37)) \cos ^{2}((0.312)(2 \pi / 62)(k-31.5))$

right eye $: w_{j k}=\cos ^{2}((0.16)(2 \pi / 62)(j-24)) \cos ^{2}((0.312)(2 \pi / 62)(k-31.5))$.

The maxima of the sensitivity distributions are located at $\left(15^{\circ}, 0^{\circ}\right)$ for the left and $\left(-15^{\circ}, 0^{\circ}\right)$ for the right eye. The time-dependent output of the model HSE cell was calculated in the following way:

$R(t)=\left(\sum_{1}^{j} \sum_{1}^{k} w_{j k}\left(E_{\mathrm{e}}^{*} g_{\mathrm{e} j k}(t)+E_{\mathrm{i}}^{*} g_{\mathrm{i} j k}(t)\right)\right) /\left(\sum_{1}^{j} \sum_{1}^{k}\left(w_{j k}\left(g_{\mathrm{e} j k}(t)+g_{\mathrm{i} j k}(t)\right)+g_{0}\right)\right)$.

Since membrane conductances cannot assume negative values, $g_{\mathrm{e}}$ and $g_{\mathrm{i}}$ were set to zero if the output of the corresponding detector subunits was smaller than zero. Based on previous combined electrophysiological and model studies on fly motion detection (Egelhaaf et al 1989), an asymmetry between excitatory and inhibitory reversal potential was assumed with 
$\left|E_{\mathrm{i}}\right|=\alpha^{*}\left|E_{\mathrm{e}}\right|, \alpha<1$. Equation (1) describes the output of the model cell in accordance with electrophysiological findings in HS cells (Borst et al 1995).

The different model parameters were determined by varying them systematically in small steps and by comparing the resulting model responses with the corresponding neuronal responses. The parameter adjustment was made separately for two different behaviourally generated motion sequences by minimizing the summed squared difference between the neuronal and the model responses. To calculate the error values, the model responses had to be scaled. The scaling factor for the model response amounts to $\left(\sigma_{\text {cell }-l}+\sigma_{\text {cell }-r}\right) /\left(\sigma_{\text {model }-l}+\right.$ $\sigma_{\text {model }-r}$ ) with $\sigma_{\text {cell }-l}$ and $\sigma_{\text {cell }-r}$ being the standard deviations across time of the left and right cell responses and $\sigma_{\text {model }-l}$ and $\sigma_{\text {model }-r}$ the standard deviations across time of the corresponding left and right model cell responses, respectively. The smallest error was obtained for both image sequences with almost the same parameter settings. Therefore all model simulations were done with the corresponding average parameters: time constant for high-pass filter, $35 \mathrm{~ms}$; time constant for low-pass filter, $150 \mathrm{~ms} ; \alpha=0.975 ; g_{0}=125000$. The brightness values of each pixel of the stimulus pattern ranged between zero and 255 . As for the electrophysiological data, the reference level of the model responses was determined as the mean response during a $250 \mathrm{~ms}$ time interval starting $750 \mathrm{~ms}$ after presentation of the first stationary image.

The similarity index for the time course of the model responses to different motion sequences was calculated in a slightly different way from the similarity index for the corresponding experimental data, because our model is entirely deterministic and thus there is no variability in the model response. The model responses to the two stimuli were crosscorrelated and normalized to the autocorrelograms of the two corresponding response traces. The similarity in the overall amplitude of the model responses was calculated in the same way as was done for the corresponding experimental data (see above).

\section{Results}

An exemplary response of the right-hand HSE cell to optic flow as experienced by a walking fly is shown in figure 3 together with the corresponding response of the model cell. Even while walking on a relatively straight section of the track (figure 3(B)), the fly continually changed the direction of its longitudinal body axis. These changes go along with permanent modulations of the fly's angular velocity (figure 3(A)). As a consequence, the membrane potentials of both the HSE cell and its model equivalent strongly fluctuate around the reference level (figures 3(C) and (D)), following to some extent the modulations of the angular velocity. It should be noted that the HSE cell responds to motion with pronounced graded de- and hyperpolarizations even when recorded close to the output terminal (Hausen 1982a). Despite differences in the fine structure of the time course of the HSE-cell response and the model response, both the cell and its model equivalent exhibit basically the same relationship between the instantaneous velocity and response amplitude. Whereas for relatively small angular velocities (below approximately $30^{\circ} \mathrm{s}^{-1}$ ) the response amplitude increases greatly with increasing velocity, it no longer increases if the velocity increases beyond this range (figures $3(\mathrm{E})$ and $(\mathrm{F})$ ). At high angular velocities the response amplitude appears to decrease again. This decrease is less pronounced in the HSE cell as compared to the model cell (compare figures 3(E) and (F)). Since walking flies frequently reach angular velocities of up to $500^{\circ} \mathrm{s}^{-1}$, this finding suggests that, irrespective of the slight differences between cellular and model responses, most of the range of angular velocities generated by walking flies is represented by only a small part of the operating range of the HSE cell and the model cell, respectively. Although the angular velocity distribution of walking flies has a single peak (figure $3(\mathrm{G})$ ), the distributions of the corresponding response amplitudes of both the HSE cell and the model show two distinct peaks at large deviations of 

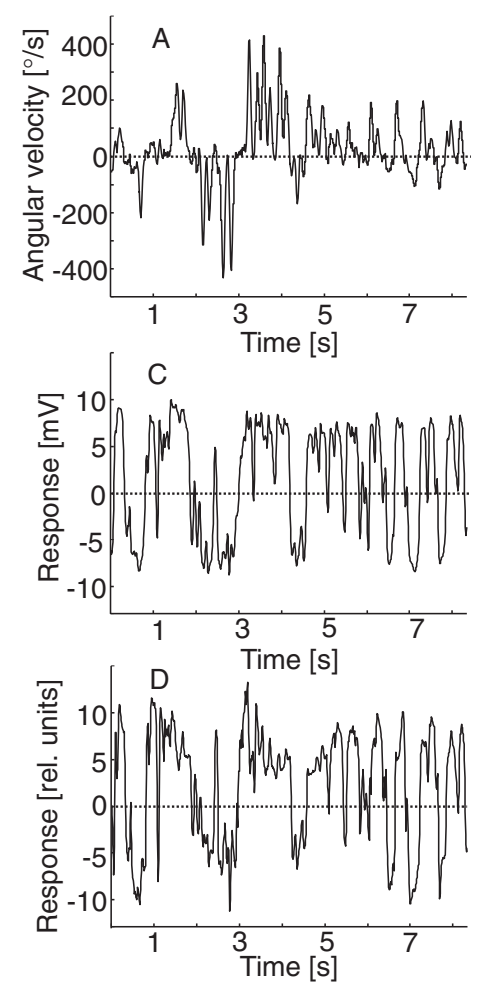

$\mathrm{B}$
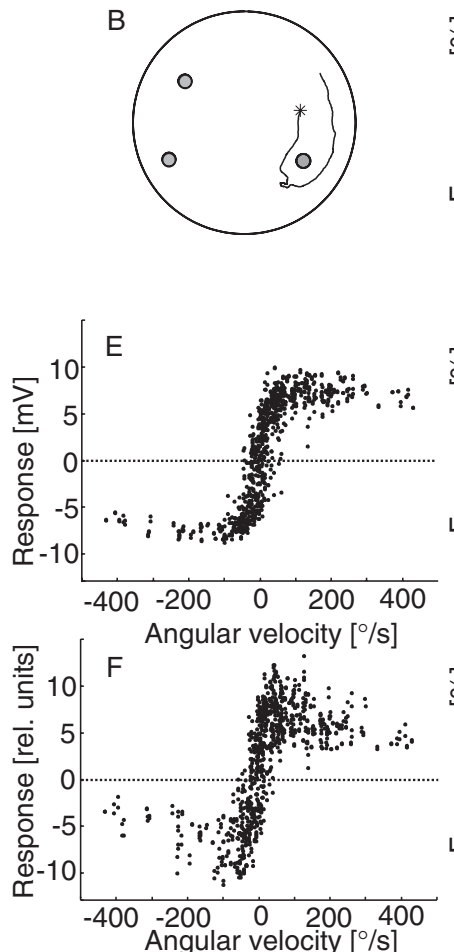

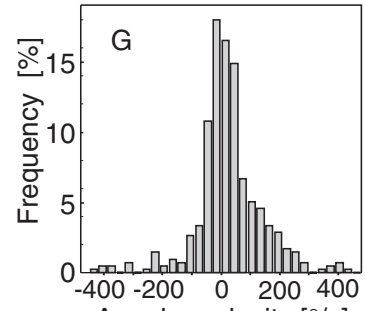

Angular velocity $[\%$ s]
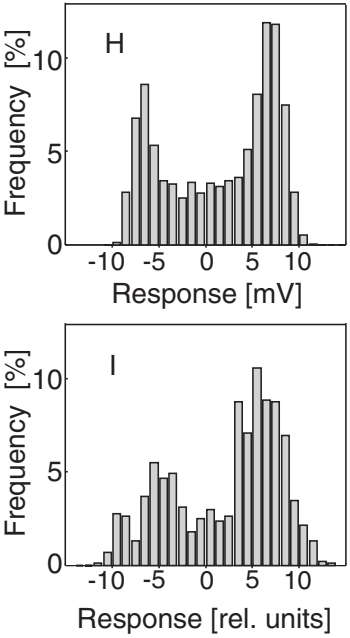

Figure 3. Responses of the HSE and its model equivalent to behaviourally generated optic flow. (A) Turning velocity of the fly while walking on a track in a textured arena containing three cylindrical objects (see (B)). The dotted horizontal line indicates $0^{\circ} \mathrm{s}^{-1}$. (B) Schematic bird eye view of the arena (diameter $0.5 \mathrm{~m}$; height $0.3 \mathrm{~m}$ ), the walking track and the position of objects (for texture see figure 1(A)). (C) Time-dependent average response of eight HSE cells to the image sequence corresponding to the track shown in (B). The average response was smoothed by a running average (width, $30 \mathrm{~ms}$ ). The sampling rate was $2 \mathrm{kHz}$. The dotted horizontal line indicates the resting potential as obtained during the $250 \mathrm{~ms}$ period prior to motion onset. (D) Time-dependent response of the output cell of the model to the image sequence corresponding to the track shown in (B). The dotted horizontal line indicates the model output as obtained without motion stimulation. (E) The response amplitude of the HSE cell as a function of turning velocity (temporal resolution $10 \mathrm{~ms}$ ). The time-dependent angular velocity and the average response trace were shifted with respect to each other by $30 \mathrm{~ms}$ before plotting. The dotted horizontal line indicates the resting potential as obtained during the $250 \mathrm{~ms}$ period prior to motion onset. $(\mathrm{F})$ The response amplitude of the model output cell as a function of turning velocity (temporal resolution $10 \mathrm{~ms}$ ). The time-dependent angular velocity and the average response trace were shifted with respect to each other by $10 \mathrm{~ms}$ before plotting. The dotted horizontal line indicates the model output as obtained without motion stimulation. (G) Distribution of angular velocties of the fly on the walking track shown in (B). The frequencies of angular velocities were determined in classes of width $30^{\circ} \mathrm{s}^{-1}$. (H) Distribution of response levels of the HSE cell when stimulated with motion sequences as seen on the track shown in (B). The frequency of response levels was determined within classes of width $1 \mathrm{mV}$. (I) Distribution of response levels of the output cell of the model when stimulated with motion sequences as seen on the track shown in (B). The frequency of response levels was determined within classes of width 1 relative unit. The experimental data are taken from the article by Kern et al (2001).

the response from the reference level (figures $3(\mathrm{H})$ and $(\mathrm{I})$ ). In the model these two peaks are less pronounced than in the HSE cell. Nonetheless, for optic flow generated by walking flies, both the HSE cell and the model perform like a kind of flip-flop. This characteristic feature is 

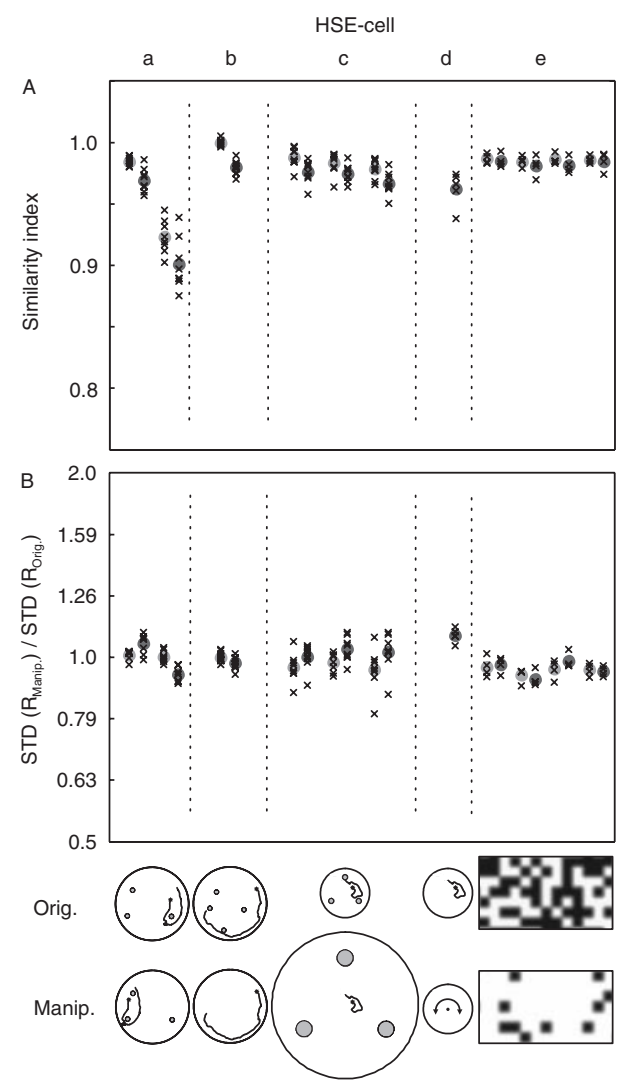

Figure 4. Similarity of responses of the HSE cell to various original optic flow stimuli and to manipulated versions of them. (A) Similarity of time courses. For the definition of the similarity index see section 2. A similarity index of unity means that the time courses of individual responses obtained under the two different stimulus conditions are as similar as the time courses of individual responses obtained under the same stimulus condition. (B) Similarity of response amplitudes, i.e. standard deviation (STD) over time (see section 2). A value of unity means that responses to manipulated and the corresponding original stimulus fluctuate with the same standard deviation. Note the logarithmic scale of the $y$-axis. For each pair of original and manipulated stimulus conditions, the left-hand data points refer to the responses of the left-hand HSE cells and the righthand data points refer to the right-hand cells (crosses, results for individual cells; filled circles, mean results). Some of the manipulations are illustrated in the insets. Filled circles within insets denote the position and diameter of objects in the arena. (a) Similarity of responses to the track in its original position versus the track displaced to the centre of the arena (left), and the track in its original position versus the track displaced to the opposite side of the arena (right). Arena diameter, $0.5 \mathrm{~m}$; height, $0.3 \mathrm{~m}$; for texture see figure 1(A). (b) Four objects present during the original walk of the fly were removed (same arena as in (a)). (c) An arena (diameter $0.31 \mathrm{~m}$, height $0.3 \mathrm{~m} ; 50 \%$ black and white texture, see inset of (e), upper pattern) was enlarged by a factor of 1.5, 2.0 and 3.0 (data from left to right). The enlargement includes the objects as well as the pattern on the arena wall and on the objects. The position of the track with respect to the arena centre was kept the same. (d) The translational component of the original walking track was eliminated and the fly rotated around the arena centre. No objects were present in the arena. Arena pattern as for (c). (e) The original $50 \%$ black and white texture was exchanged for a texture with only $12 \%$ black elements. This was done and tested for the originally sized arena (diameter $0.31 \mathrm{~m}$, height $0.3 \mathrm{~m}$ ) as well as for the arena enlarged by a factor of 1.5, 2.0 and 3.0 (data shown from left to right). The texture density of the patterns covering the objects was kept as in the originally sized arena. The enlargement of the arena includes the pattern on the arena wall and on the objects. The track was the same as in (c). The number of cells was eight (a), ten (b), eight (c), five (d) and four (e); the total number of individual response traces was 237 (a), 132 (b), 177 (c), 120 (d) and 148 (e). The data in (A) obtained for the right-hand HSE cell are taken from Kern et al (2001). 

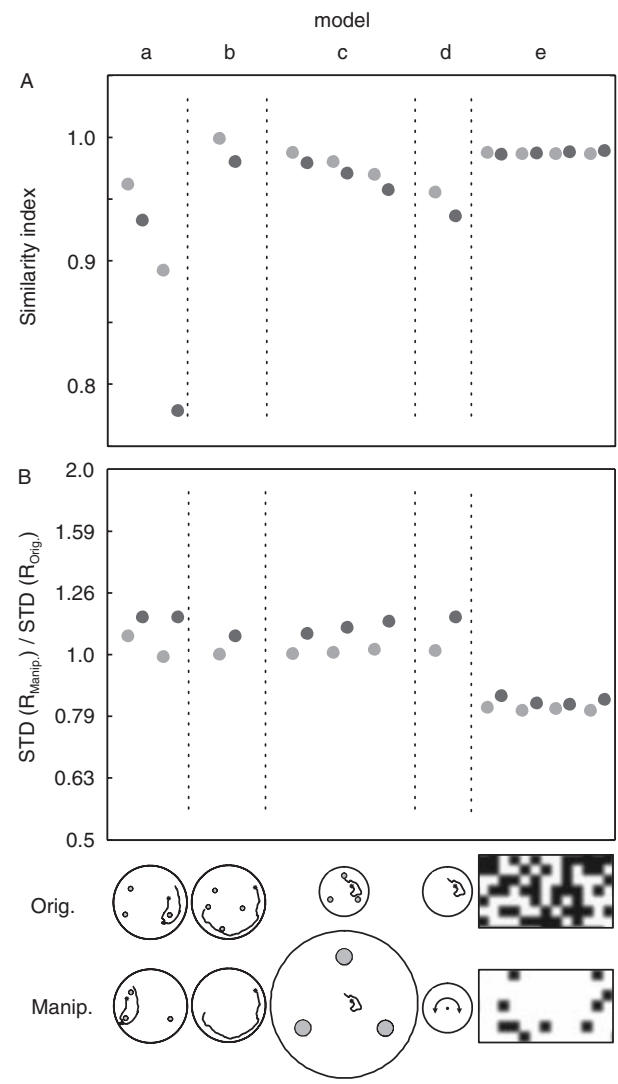

Figure 5. Similarity of the responses of the model HSE cell to various original optic flow stimuli and to manipulated versions of them. (A) Similarity of time courses. For the definition of the similarity index see section 2. A similarity index of unity means that the responses obtained under the two different stimulus conditions are identical. (B) Similarity of response amplitudes, i.e. STD over time (see section 2). A value of unity means that the fluctuations of the responses to the manipulated and the original stimulus have the same standard deviation. Note the logarithmic scale of the $y$ axis. For each pair of original and manipulated stimulus conditions, the left-hand data points refer to the responses of the left-hand model HSE cell; the right-hand data points refer to the right-hand model cells. For explanation of the insets see figure 4 .

obtained for the right and the left HSE cells as well as for the other behaviourally generated motion sequences that were used as visual stimuli (data not shown). In conclusion, while the fly is walking the HSE cell and its model equivalent do not encode the angular velocity of the animal faithfully. Rather they appear to encode the turning direction largely independent of the velocity of the retinal images. This independence is more pronounced in the real than in the model neuron.

While walking on its track (figure 3(B)), the distance from the fly's eyes to the arena wall and to the objects changes considerably. Hence, the translational optic flow component as well as the textural properties of the retinal input change during the different phases of a given walking track. To test the influence of the layout of the environment on the neuronal and the model responses, we manipulated the original image sequences as experienced by the fly on its track. These manipulations included displacements of the track in the arena and removing objects from the arena as well as changing the arena size and the texture on its wall. In this way the textural properties of the retinal input as well as the translational component of the optic flow were altered while the rotational optic flow component was kept constant. The similarity between the time courses of the responses to the original and to the corresponding manipulated version was determined by cross-correlating the responses to both types of optic flow and by appropriate normalization (see section 2).

Displacing the walking track within the arena did not much alter the time course of either the neuronal or the model response (figures 4(A)(a), 5(A)(a)). Only in one extreme case when the fly was very close to the wall in one section of the track was there a clear difference 
between the responses to the original and to the manipulated optic flow (see Kern et al 2001). This difference was more pronounced in the model responses. Removing the objects which were present in the arena during the behavioural experiment before reconstructing the image sequences did not much alter the time course of the responses (figures $4(\mathrm{~A})(\mathrm{b}), 5(\mathrm{~A})(\mathrm{b})$ ). The neuronal and the model responses did not much change on increasing the arena size and, thus, on decreasing the translational optic flow component (figures $4(\mathrm{~A})(\mathrm{c})$ and $5(\mathrm{~A})(\mathrm{c})$ ). This finding also holds when eliminating the translational optic flow component entirely by rotating the fly in the centre of the arena at the original turning velocities. Also then the responses to the modified optic flow are very similar to those obtained on the original walking track in a small arena (figures $4(\mathrm{~A})(\mathrm{d})$ and $5(\mathrm{~A})(\mathrm{d})$ ). To appreciate the significance of this finding, it should be noted that the HSE cell strongly responds to the translational component of optic flow when it is stimulated with optic flow experienced on artificial walking paths, such as while translating on a straight course in the arena (Kern et al 2001). Furthermore, the responses of the HSE cell and of the model cell are rather robust to changes in the density of texture elements on the wall of the arena (figures 4(A)(e) and 5(A)(e)).

Not only the time course of both the neuronal and the model responses, but also their amplitude, were rather robust with respect to all tested manipulations of the optic flow. The ratio of the standard deviations over time between the average responses to the manipulated and to the original optic flow (see section 2) ranged between 0.9 and 1.1 for the HSE cell (figure 4(B)) and between 0.8 and 1.2 for the model cell (figure 5(B)). This finding indicates very similar amplitudes of the responses to manipulated and original image sequences.

It is obvious from the data shown in figure 4 that the similarity of the responses to the original and to the manipulated optic flow is slightly larger for the left than for the right HSE cell. One reason for this difference might be systematic differences of the retinal input of the two eyes in the specific situations that were tested. For instance, on the walking track where the objects were removed from the manipulated image sequence the left eye views the objects on the original walking track only for a short instance of time, whereas the right eye saw the objects for most of the time. The left HSE cell should thus be less affected by removing these objects than the right HSE cell. Despite these differences in detail, the results displayed in figure 4 corroborate the above conclusion that the HSE cell encodes the direction of turns largely independent of the three-dimensional layout of the environment and its textural properties when it is stimulated with optic flow as experienced by walking flies. Moreover, the model simulations reveal that our model of the fly motion pathway can account, at least qualitatively, for the main features of the experimentally determined responses to naturalistic optic flow (compare figures 4 and 5). The model can thus explain the ability of the HSE cell to encode the directions of turns largely independent of the spatial layout of the surroundings and the textural properties of the environment.

\section{Discussion}

Apart from extreme situations, the fly HSE cell extracts the direction of turns from complex optic flow generated by freely walking animals. The direction of turns is extracted largely independent of the three-dimensional layout and of the textural properties of the environment. This finding is remarkable, given the highly ambiguous responses of the HSE cell when stimulated with simple approximations of optic flow and its pronounced responses to purely translational optic flow (see section 1). The main features of the responses of the HSE cell to behaviourally generated optic flow can be explained in a first approximation by a simple network model of the fly's visual motion pathway that was originally proposed on the basis of neuronal responses to simple motion stimuli (see section 2). A similarly high specificity 
for behaviourally generated visual input as found for the HSE cell has been shown to exist in another motion sensitive neuron of the fly, i.e. in one of the so-called figure detection (FD) cells (Kimmerle and Egelhaaf 2000). This cell was found to be more specific to moving objects when stimulated with optic flow that was generated while the fly executed a behavioural fixation task as compared with simple object and background motion. However, in contrast to our present behavioural experiments, the latter experiments were done on tethered flies flying in a flight simulator.

To understand the significance of our results obtained with behaviourally generated optic flow three questions should be addressed. (i) To what extent do neuronal responses in an experimental situation suitable for electrophysiological analysis reflect the state of the animal in an unrestrained behavioural situation? (ii) Why does behaviourally generated optic flow lead to seemingly different conclusions from simple motion stimuli? (iii) What are the functional implications of the relative invariance of the neuronal responses with respect to environmental properties?

\subsection{Replay of behaviourally generated optic flow}

Can neuronal responses to behaviourally generated optic flow be regarded as equivalent to neuronal responses in the corresponding behavioural situation? Apart from the fact that the animal is inevitably restrained and partly dissected in electrophysiological experiments, it also has no chance to influence the visual input by its reactions ('open-loop conditions') as is the case in behavioural situations ('closed-loop conditions'). Indeed, replaying behaviourally generated optic flow to the tethered flying fly in a flight simulator under open-loop conditions induces weaker responses than in the preceding closed-loop experiments (Heisenberg and Wolf 1988). However, there is good evidence that this difference between open- and closed-loop performance is attributable to signal processing subsequent to the motion-sensitive TCs in the fly's third visual neuropile. (i) The responses of a motion sensitive TC recorded in an electrophysiological situation do not differ from the responses recorded extracellularly in a tethered flying animal (Heide 1983). (ii) The response variability of TCs is much smaller than the behavioural variability. For instance, in model simulations a considerable amount of variability had to be introduced to the output of the TCs to simulate realistic optomotor behaviour in the fly (Warzecha and Egelhaaf 1996). Moreover, behavioural responses to visual stimuli are affected by 'attentional' influences in a way that has never been observed at the level of the motion sensitive neurons (Wolf and Heisenberg 1980, Kern et al unpublished results). (iii) So far there is no evidence that the responses of TCs to visual motion are influenced by other sensory modalities. For all these reasons, the responses of the HSE cell and of other TCs recorded under replay conditions are considered to be indicative of the responses of these cells in behavioural situations.

\subsection{Specificity of neuronal responses under naturalistic and simple stimulus conditions}

Why does the HSE cell extract the direction of self-motion of the animal from behaviourally generated optic flow largely unaffected by the environmental properties and thus more specifically than was expected from the responses to simple stimuli? The peculiar dynamical properties of behaviourally generated visual input might be one reason. This is suggested by the different specificity of the HSE cell for the rotational flow component as found in responses to optic flow generated on simple artificial walking tracks, on the one hand, and to optic flow that was generated by the animal itself, on the other hand (Kern et al 2001). The nonlinearities inherent in the mechanisms underlying the computation of visual motion information (for 
reviews see Egelhaaf and Borst 1993b, Borst et al 1995, Egelhaaf and Warzecha 1999) might be another reason why the responses of both the HSE cell and of the model cell are almost invariant with respect to the layout of the environment. This invariance is only observed if the retinal images are characterized by a broad spatial frequency spectrum rather than by only one frequency component (Kern, unpublished experimental results; see also Dror et al 2001). The spatial frequency content of the stimulus patterns we used in our experiments can be roughly approximated by an $1 / f$ function, with $f$ representing the spatial frequency. The spatial frequency content of natural scenes has been shown to be characterized by similar functional relationships (Schaaf and Hateren 1996).

The responses of TCs are not exclusively determined by the current stimulus conditions, but are also affected by adaptational processes (Harris et al 2000, Kurtz et al 2000, Harris et al 1999, Borst and Egelhaaf 1987, Ruyter van Steveninck et al 1986, Maddess and Laughlin 1985). Nonetheless, we could account for the qualitative features of our present experimental results by a model that does not contain any adaptive process. Hence, motion adaptation is not essential for explaining the ability of the HSE cell to extract the direction of the fly's turns from behaviourally generated optic flow largely independent of the spatial layout and the textural properties of the environment. This conclusion is not meant to imply that motion adaptation is irrelevant. Motion adaptation may rather play a more subtle role in optic flow processing in walking flies than could be unravelled by our current analysis. Moreover, it might be important in different behavioural contexts, such as during flight.

There are two nonlinearities in the model of the fly's visual motion pathway which appear to be decisive for simulating our experimental results, (i) the multiplicative interaction between signals originating from neighbouring points in visual space, which is characteristic of the correlation-type movement detectors as implemented in our model, and (ii) the spatial integration properties of the output neuron of the model circuit, which is meant to mimic the HSE cell.

As a consequence of their specific nonlinear computations, the responses of correlationtype movement detectors and of the motion detection system of the fly do not only depend on pattern velocity. Even the steady-state responses to constant motion depend, apart from velocity, on the textural properties and contrast of the stimulus pattern (for a review see Egelhaaf and Borst 1993b). Moreover, it has been shown theoretically as well as experimentally that the time course of the spatially integrated output of local movement detectors depends not only on pattern velocity but also on acceleration and even higher temporal derivatives (Egelhaaf and Reichardt 1987). This implies that the time course of the spatially integrated detector response reflects the time course of pattern velocity only within a limited dynamic range, i.e. when the pattern velocity and its changes changes are sufficiently small (see also figure 3 ). In this dynamic range the pattern velocity can be estimated on the basis of the time-dependent responses of TCs (Haag and Borst 1997, Bialek et al 1991). At larger velocities and velocity changes, the time course of pattern velocity can be reconstructed much less accurately (Haag and Borst 1997), because the response is then determined to an increasing extent by the temporal derivatives of pattern velocity (Egelhaaf and Reichardt 1987). In this dynamic range, the peak amplitude of the time-dependent responses stays almost constant and only the shape of the response profile changes with changing dynamics of the motion stimulus (Egelhaaf and Reichardt 1987). The flip-flop-like behaviour of the HSE cell when stimulated with behaviourally generated optic flow (see figures $3(\mathrm{E})$ and $(\mathrm{H})$ ) can be explained, at least to some extent, on this basis. It does not represent a consequence of saturation nonlinearities at the level of the HSE cell, although the latter may shape the temporal fine structure of the responses (see below), but is essentially the consequence of the mechanisms underlying local motion detection. 
In accordance with our findings it was shown in a recent study (Brenner et al 2000) that, in a certain dynamic range of motion stimulation, the peak response amplitude of a fly motion sensitive TC (the H1-cell) changes only relatively little when the standard deviation of white-noise velocity fluctuations is increased. This result was interpreted as a consequence of adaptive processes in the fly's motion pathway, which are assumed to rescale the input/output relation (Brenner et al 2000). In contrast to this interpretation, our model simulations indicate that the near invariance of the response amplitude described by Brenner et al (2000) can be explained in a more parsimonious way as an emergent property of the computations underlying motion detection.

The nonlinear spatial integration properties of the output cell of the model are indicated by preliminary model simulations to be essential for explaining the experimentally established virtual independence of the HSE-cell responses from texture density. When the output cell of the model is configured to spatially integrate its retinotopic input linearly the response amplitude depends much more on the texture density than when the nonlinear spatial integration properties of the HSE cell are taken into account in the model (Lutterklas et al unpublished results). It should be noted that the characteristic nonlinear spatial integration properties of the fly's visual motion pathway are more sophisticated than a simple output saturation. This is because TCs such as the HSE cell receive opponent input from excitatory and inhibitory input channels. Since the ratio of activation of both types of input depends on the direction of motion and on stimulus velocity, the HSE cell as well the output neuron of the model approach different saturation levels for different velocities (Single et al 1997, Borst et al 1995, Egelhaaf and Borst 1993a). This gain control might lead to response levels that are independent of textural changes in the environment as may frequently happen to the fly under outdoors conditions.

For the gain control of the model to operate appropriately the input of the local motion detectors needs to represent mainly changes in light intensity rather than the absolute light intensity itself. In our model this is accomplished by the high-pass filter in the input lines of the local motion detectors. Without this filtering, the input synapses of the output cell of the model are tonically active even if they are not stimulated by visual motion. All inputs would then contribute considerably to the input conductance of the output cell irrespective of whether or not they are driven by motion stimulation. As a consequence the input conductance would be modulated only a little about its large mean value during motion stimulation. Hence, if the mean light intensity were represented strongly at the movement detector output, the input conductance of the model output cell (corresponding to the denominator in equation (1)) would be largely independent of the number of inputs activated by visual motion. Nonetheless, as a consequence of the opponent input of the model output cell, the response amplitude would increase greatly with an increasing number of retinotopic inputs activated by motion and thus with increasing pattern density (see the numerator in equation (1)). These considerations imply that the input of the movement detectors is not much affected by the mean luminance. Indeed, there is experimental evidence that the response amplitude of a fly TC is not signficantly different if determined outdoors on a sunny day or during dusk at light levels that are smaller by five orders of magnitude (Egelhaaf et al 2001).

\subsection{Functional aspects}

It was concluded above that, at least in walking flies, the motion vision system operates in the nonlinear range of its velocity/response relationship. By reducing the time constant of the local movement detectors it would have been possible, at least in principle, to adjust the operating range of the motion detection system so that the time course of behaviourally generated optic flow is signalled about linearly. Is there any computational reason why this 
has not evolved? We are currently addressing this question by systematic model simulations. At the moment we can only speculate about the possible reasons. One reason might have its basis in the noisiness of neuronal responses (for a review see Warzecha and Egelhaaf 2001). Given the limited response range of a neuron, information is transmitted most efficiently, at least from an information theoretical point of view, when all response levels are used equally often (Shannon and Weaver 1949). This condition is realized when the function relating the response amplitude to the input level is matched to the statistical distribution of stimulus levels. This kind of matched coding was concluded to operate in the peripheral visual system of the fly (for a review see Laughlin 1994) as well as at the level of TCs (Brenner et al 2000). In contrast to the conclusion obtained for a fly TC when stimulated with random velocity fluctuations, our results indicate that matched coding cannot be the reason for the peculiar performance of the HSE cell under naturalistic stimulus conditions. This is because the responses of the HSE cell elicited by behaviourally generated optic flow tend to assume preferentially two response levels rather than all activity levels with about the same frequency (see figure $3(\mathrm{H})$ ). Hence, conclusions about the coding properties of neuronal circuits are of only limited value if they are based on stimuli exclusively designed by the experimenters rather than on what the animal really sees in behavioural situations.

Our experimental data and modelling results suggest that the visual motion pathway operates under behavioural conditions (at least in walking flies) in a range where it does not encode pattern velocity linearly, but basically switches between two states and, thus, appears to encode 'only' the direction of the fly's turns. It is suggested that only in this dynamic range, rather than in the linear range, the system is relatively invariant against changes in the textural properties of the environment. This hypothesis, however, still needs to be tested more rigorously than has been done so far. In any case, the relative invariance of the responses of the HSE cell with respect to the environmental properties is likely to be particularly relevant in more natural environments than the laboratory setting where our behavioural experiments have been done so far. This is because under outdoors conditions the statistical properties of the retinal input might be more heterogeneous during a behavioural sequence than in the laboratory.

Although the responses to behaviourally generated optic flow are rather invariant against environmental changes, this invariance is far from being complete. For instance, when the fly is displaced very close to the wall, the response may deviate considerably from those obtained at somewhat larger distances (Kern et al 2001). We are currently investigating whether these differences in the responses may provide useful information about the spatial layout of the environment. According to this possibility the responses are invariant against changes in the spatial layout of the environment as long as the fly is sufficiently apart from obstacles and thus does not have to care much about them in order to prevent a collision. Only if the fly is very close to obstacles does it have to care about them, and here the difference signal of the outputs of the two HSE cells may indicate this fact and collisions can be prevented by changing the course of locomotion. Although this idea might be appealing, it needs to be tested carefully.

The neuronal representation of optic flow of freely moving flies has been analysed, so far, on walking animals. Despite their name, flies spend a large proportion of their time walking around in their environment (Dethier 1976). Hence, walking may be as important for a fly as flying, although in different behavioural contexts. Since for methodological reasons optic flow experienced by flying flies could not yet be replayed in electrophysiological experiments, it is currently not possible to assess to what extent our conclusions obtained for walking flies generalize to free flight. This important point will be approached with a much faster stimulation set-up, which is currently being developed. 
The HSE cell is just one element, though an important one, of an ensemble of some tens of output neurons of the fly's visual motion pathway. From their responses determined with simple stimuli these neurons are expected to encode other aspects of optic flow than the HSE cell (see e.g. Krapp 2000, Hausen and Egelhaaf 1989). It will only be possible to understand what aspects of self-motion are extracted under normal behavioural conditions if the responses of this whole ensemble of cells to behaviourally generated optic flow is understood. Current experiments in our laboratory are heading into this direction.

The outcome of the present analysis stresses the importance of analysing the performance of neuronal circuits also under naturalistic operating conditions. Only then will it be possible to understand how the mechanisms underlying visual information processing are adapted to the natural operating conditions of the system.

\section{Acknowledgments}

We are much indebted to A-K Warzecha who wrote the program for data acquisition. We would like to thank N Böddeker, R Kurtz, K Karmeier and A-K Warzecha for valuable discussions and suggestions on the manuscript. This work was supported by the DFG.

\section{References}

Baker C L 1990 Spatial- and temporal-frequency selectivity as a basis for velocity preference in cat striate cortex neurons Vis. Neurosci. 4 101-13

Bialek W, Rieke F, Ruyter van Steveninck R d and Warland D 1991 Reading a neural code Science 252 1854-7

Borst A and Egelhaaf M 1987 Temporal modulation of luminance adapts time constant of fly movement detectors Biol. Cybern. 56 209-15

Borst A, Egelhaaf M and Haag J 1995 Mechanisms of dendritic integration underlying gain control in fly motionsensitive interneurons J. Comput. Neurosci. 2 5-18

Brenner N, Bialek W and Ruyter van Steveninck R d 2000 Adaptive rescaling maximizes information transmission Neuron 26 695-702

Cassanello C R, Priebe N J and Lisberger S G 2000 The speed tuning of single units in macaque visual area MT depends upon spatial form Soc. Neurosci. Abstr. 1673

Dahmen H J, Wüst R M and Zeil J 1997 Extracting egomotion parameters from optic flow: principal limits for animals and machines From Living Eyes to Seeing Machines ed M V Srinivasan and S Venkatesh (Oxford: Oxford University Press) pp 174-98

Dethier V G 1976 The Hungry Fly. A Physiological Study of the Behavior Associated with Feeding (Cambridge, MA: Harvard University Press)

Dror R O, O'Carroll D C and Laughlin S B 2001 Accuracy of velocity estimation by Reichardt correlators J. Opt. Soc. Am. A 18 241-52

Eckert H 1980 Functional properties of the H1-neurone in the third optic ganglion of the blowfly Phaenicia J. Comp. Physiol. 135 29-39

Egelhaaf M and Borst A 1993a A look into the cockpit of the fly: visual orientation, algorithms, and identified neurons J. Neurosci. 13 4563-74

-1993b Movement detection in arthropods Visual Motion and its Role in the Stabilization of Gaze ed J Wallman and F A Miles (Amsterdam: Elsevier) pp 53-77

Egelhaaf M, Borst A and Reichardt W 1989 Computational structure of a biological motion detection system as revealed by local detector analysis in the fly's nervous system J. Opt. Soc. Am. A 6 1070-87

Egelhaaf M, Grewe J, Kern R and Warzecha A-K 2001 Outdoor performance of a motion-sensitive neuron in the blowfly Vision Res. at press

Egelhaaf M, Hausen K, Reichardt W and Wehrhahn C 1988 Visual course control in flies relies on neuronal computation of object and background motion Trends Neurosci. 11 351-8

Egelhaaf M and Reichardt W 1987 Dynamic response properties of movement detectors: theoretical analysis and electrophysiological investigation in the visual system of the fly Biol. Cybern. 56 69-87

Egelhaaf M and Warzecha A-K 1999 Encoding of motion in real time by the fly visual system Curr. Opin. Neurobiol. $9454-60$

Gibson J J 1979 The Ecological Approach to Visual Perception (Boston: Houghton Mifflin) 
Haag J and Borst A 1997 Encoding of visual motion information and reliability in spiking and graded potential neurons J. Neurosci. 17 4809-19

Harris R A, O'Carroll D C and Laughlin S B 1999 Adaptation and the temporal delay filter of fly motion detectors Vision Res. 39 2603-13

-2000 Contrast gain reduction in fly motion adaptation Neuron 28 595-606

Hausen K 1982a Motion sensitive interneurons in the optomotor system of the fly I. The horizontal cells: structure and signals Biol. Cybern. 45 143-56

1982b Motion sensitive interneurons in the optomotor system of the fly. II. The horizontal cells: receptive field organization and response characteristics Biol. Cybern. 46 67-79

Hausen K and Egelhaaf M 1989 Neural mechanisms of visual course control in insects Facets of Vision ed D Stavenga and R Hardie (Berlin: Springer) pp 391-424

Heide G 1983 Neural mechanisms of flight control in diptera BIONA Report ed W Nachtigall (Mainz, Stuttgart: Akademie der Wissenschaften und der Literatur zu Mainz-Fischer) pp 35-52

Heisenberg M and Wolf R 1988 Reafferent control of optomotor yaw torque in Drosophila Melanogaster J. Comp. Physiol. A 163 373-88

Horstmann W, Egelhaaf M and Warzecha A-K 2000 Synaptic interactions increase optic flow specificity Eur. $J$. Neurosci. 12 2157-65

Juusola M, French A S, Uusitalo R O and Weckström M 1996 Information processing by graded-potential transmission through tonically active synapses Trends Neurosci. $19292-7$

Kern R and Egelhaaf M 2000 Optomotor course control in flies with largely asymmetric visual input J. Comp. Physiol. A $18645-55$

Kern R, Lutterklas M and Egelhaaf M 2000 Neural representation of optic flow experienced by unilaterally blinded flies on their mean walking trajectories J. Comp. Physiol. A 186 467-79

Kern R, Petereit C and Egelhaaf M 2001 Neural processing of naturalistic optic flow J. Neurosci. 21 1-5

Kim J-N, Mulligan K and Sherk H 1997 Simulated optic flow and extrastriate cortex. I. Optic flow versus texture $J$. Neurophysiol. 77 554-61

Kimmerle B and Egelhaaf M 2000 Performance of fly visual interneurons during object fixation $J$. Neurosci. 20 6256-66

Koenderink J J 1986 Optic flow Vision Res. 26 161-80

Kording K P, Einhauser W and König P 2000 Learning invariances from natural images Soc. Neurosci. Abstr. 26 366.17

Krapp H 2000 Neuronal matched filters for optic flow processing in flying insects Neuronal Processing of Optic Flow ed M Lappe (San Diego, CA: Academic) pp 93-120

Krapp H G, Hengstenberg R and Egelhaaf M 2001 Binocular contribution to optic flow processing in the fly visual system J. Neurophysiol. 85 724-34

Kurtz R, Dürr V and Egelhaaf M 2000 Dendritic calcium accumulation associated with direction selective adaptation in visual motion sensitive neurons in vivo J. Neurophysiol. 84 1914-23

Lappe M (ed) 2000 Neuronal Processing of Optic Flow (San Diego: Academic)

Laughlin S B 1994 Matching coding, circuits, cells, and molecules to signals: general principles of retinal design in the fly's eye Prog. Ret. Eye Res. 13 165-96

Maddess T and Laughlin S B 1985 Adaptation of the motion-sensitive neuron H1 is generated locally and governed by contrast frequency Proc. R. Soc. B 225 251-75

Miles F A and Wallman J (ed) 1993 Visual Motion and its Role in the Stabilization of Gaze (Amsterdam: Elsevier)

Mulligan K, Kim J-N and Sherk H 1997 Simulated optic flow and extrastriate cortex. II. Responses to bar versus large-field stimuli J. Neurophysiol. 77 562-70

Passaglia C, Dodge F, Herzog E, Jackson S and Barlow R 1997 Deciphering a neural code for vision Proc. Natl Acad. Sci. USA 94 12649-54

Pekel M, Lappe M, Bremmer F, Thiele A and Hoffmann K-P 1996 Neuronal responses in the motion pathway of the macaque monkey to natural optic flow stimuli Neuro Rep. $7884-8$

Pollen D A, Andrews B W and Feldon S E 1978 Spatial frequency selectivity of periodic complex cells in the visual cortex of the cat Vision Res. 18 665-82

Ruyter van Steveninck R d, Zaagman W H and Mastebroek H A K 1986 Adaptation of transient responses of a movement-sensitive neuron in the visual system of the blowfly, Calliphora erythrocephala Biol. Cybern. $\mathbf{5 4}$ $223-36$

Schaaf A v and Hateren J H v 1996 Modelling the power spectra of natural images: statistics and information Vision Res. 36 2759-70

Shannon C E and Weaver W 1949 The Mathematical Theory of Communication (Urbana, IL: University of Illinois Press) 
Single S, Haag J and Borst A 1997 Dendritic computation of direction selectivity and gain control in visual interneurons J. Neurosci. 17 6023-30

Smakman J G J, Hateren J H v and Stavenga D G 1984 Angular sensitivity of blowfly photoreceptors: Intracellular measurements and wave-optical predictions J. Comp. Physiol. A 155 239-47

Srinivasan M V, Poteser M and Kral K 1999 Motion detection in insect orientation and navigation Vision Res. 39 2749-66

Strauss R and Heisenberg M 1990 Gaze stabilizing head movements compensate for walk-induced body oscillations in the fly Drosophila melanogaster Brain-Perception-Cognition ed N Elsner and G Roth (Stuttgart: Thieme) p 63

Warzecha A-K and Egelhaaf M 1996 Intrinsic properties of biological motion detectors prevent the optomotor control system from getting unstable Phil. Trans. R. Soc. B 351 1579-1591

Wolf R and Heisenberg M 1980 On the fine structure of yaw torque in visual flight orientation of Drosophila melanogaster II. A temporally and spatially variable weighting function for the visual field ('visual attention') J. Comp. Physiol. 140 69-80

Wolf-Oberhollenzer F and Kirschfeld K 1990 Temporal frequency dependence in motion-sensitive neurons of the accessory optic system of the pigeon Naturwissenschaften 77 296-8 